\title{
A mechanical model that exhibits a gravitational critical radius
}

\author{
Kirk T. McDonald \\ Joseph Henry Laboratories, Princeton University, Princeton, NJ 08544
}

(Dec. 2, 1998)

\section{Problem}

A popular model at science museums (and also a science toy [1]) that illustrates how curvature can be associated with gravity consists of a surface of revolution $r=-k / z$ with $z<0$ about a vertical axis $z$. The curvature of the surface, combined with the vertical force of Earth's gravity, leads to an inward horizontal acceleration of $k g / r^{2}$ for a particle that slides freely on the surface in a circular, horizontal orbit.

Consider the motion of a particle that slides freely on an arbitrary surface of revolution, $r=r(z) \geq 0$, defined by a continuous and differentiable function on some interval of $z$. The surface may have a nonzero minimum radius $R$ at which the slope $d r / d z$ is infinite. Discuss the character of oscillations of the particle about circular orbits to deduce a condition that there be a critical radius $r_{\text {crit }}>R$, below which the orbits are unstable. That is, the motion of a particle with $r<r_{\text {crit }}$ rapidly leads to excursions to the minimum radius $R$, after which the particle falls off the surface.

Give one or more examples of analytic functions $r(z)$ that exhibit a critical radius as defined above. These examples provide a mechanical analogy as to how departures of gravitational curvature from that associated with a $1 / r^{2}$ force can lead to a characteristic radius inside which all motion tends toward a singularity.

\section{Solution}

We work in a cylindrical coordinate system $(r, \theta, z)$ with the $z$ axis vertical. It suffices to consider a particle of unit mass.

In the absence of friction, there is no torque on a particle about the $z$ axis, so the angular

momentum component $J=r^{2} \dot{\theta}$ about that axis is a constant of the motion, where ${ }^{\cdot}$ indicates differentiation with respect to time.

For motion on a surface of revolution $r=r(z)$, we have $\dot{r}=r^{\prime} \dot{z}$, where ' indicates differentiation with respect to $z$. Hence, the kinetic energy can be written

$$
T=\frac{1}{2}\left(\dot{r}^{2}+r^{2} \dot{\theta}^{2}+\dot{z}^{2}\right)=\frac{1}{2}\left[\dot{z}^{2}\left(1+r^{\prime 2}\right)+r^{2} \dot{\theta}^{2}\right] .
$$

The potential energy is $V=g z$. Using Lagrange's method, the equation of motion associated with the $z$ coordinate is

$$
\ddot{z}\left(1+r^{\prime 2}\right)+\dot{z}^{2} r r^{\prime \prime}=-g+\frac{J r^{\prime}}{r^{3}} .
$$

For a circular orbit at radius $r_{0}$, we have

$$
r_{0}^{3}=\frac{J^{2} r_{0}^{\prime}}{g} .
$$


We write $\dot{\theta}_{0}=\Omega$, so that $J=r_{0}^{2} \Omega$.

For a perturbation about this orbit of the form

$$
z=z_{0}+\epsilon \sin \omega t
$$

we have, to order $\epsilon$,

$$
\begin{aligned}
r(z) & \approx r\left(z_{0}\right)+r^{\prime}\left(z_{0}\right)\left(z-z_{0}\right) \\
& =r_{0}+\epsilon r_{0}^{\prime} \sin \omega t \\
r^{\prime} & \approx r_{0}^{\prime}+\epsilon r_{0}^{\prime \prime} \sin \omega t, \\
\frac{1}{r^{3}} & \approx \frac{1}{r_{0}^{3}}\left(1-3 \epsilon \sin \omega t \frac{r_{0}^{\prime}}{r_{0}}\right) .
\end{aligned}
$$

Inserting (40) into (2) and keeping terms only to order $\epsilon$, we obtain

$$
-\epsilon \omega^{2} \sin \omega t\left(1+r_{0}^{\prime 2}\right) \approx-g+\frac{J^{2}}{r_{0}^{3}}\left(r_{0}^{\prime}-3 \epsilon \sin \omega t \frac{r_{0}^{\prime 2}}{r_{0}}+\epsilon \sin \omega t r_{0}^{\prime \prime}\right) .
$$

From the zeroeth-order terms we recover (3), and from the order- $\epsilon$ terms we find that

$$
\omega^{2}=\Omega^{2} \frac{3 r_{0}^{\prime 2}-r_{0} r_{0}^{\prime \prime}}{1+r_{0}^{\prime 2}} .
$$

The orbit is unstable when $\omega^{2}<0$, i.e., when

$$
r_{0} r_{0}^{\prime \prime}>3 r_{0}^{\prime 2}
$$

This condition has the interesting geometrical interpretation (noted by a referee) that the orbit is unstable wherever

$$
\left(1 / r^{2}\right)^{\prime \prime}<0
$$

i.e., where the function $1 / r^{2}$ is concave inwards.

For example, if $r=-k / z$, then $1 / r^{2}=z^{2} / k^{2}$ is concave outwards, $\omega^{2}=J^{2} /\left(k^{2}+r_{0}^{4}\right)$, and there is no regime of instability.

We give three examples of surfaces of revolution that satisfy condition (11).

First, the hyperboloid of revolution defined by

$$
r^{2}-z^{2}=R^{2}
$$

where $R$ is a constant. Here, $r_{0}^{\prime}=z_{0} / r_{0}, r_{0}^{\prime \prime}=R^{2} / r_{0}^{3}$, and

$$
\omega^{2}=\Omega^{2} \frac{3 z_{0}^{2}-R^{2}}{2 z_{0}^{2}+R^{2}}=\Omega^{2} \frac{3 r_{0}^{2}-4 R^{2}}{2 r_{0}^{2}-R^{2}} .
$$

The orbits are unstable for

$$
z_{0}<\sqrt{3} R
$$

or equivalently, for

$$
r_{0}<\frac{2 \sqrt{3}}{3} R=1.1547 R \equiv r_{\text {crit }} .
$$


As $r_{0}$ approaches $R$, the instability growth time approaches an orbital period.

Another example is the Gaussian surface of revolution,

$$
r^{2}=R^{2} e^{z^{2}}
$$

which has a minimum radius $R$, and a critical radius $r_{\text {crit }}=R \sqrt[4]{e}=1.28 R$.

Our final example is the surface

$$
r=-\frac{k}{z \sqrt{1-z^{2}}}, \quad(-1<z<0),
$$

which has a minimum radius of $R=2 k$, approaches the surface $r=-k / z$ at large $r$ (small $z)$, and has a critical radius of $r_{\text {crit }}=6 k / \sqrt{5}=1.34 R$.

These examples arise in a $2+1$ geometry with curved space but flat time. As such, they are not fully analagous to black holes in $3+1$ geometry with both curved space and curved time. Still, they provide a glimpse as to how a particle in curved spacetime can undergo considerably more complex motion than in flat spacetime.

\section{Acknowledgement}

The author wishes to thank Ori Ganor and Vipul Periwal for discussions of this problem.

\section{References}

[1] The $\operatorname{Vortx}(\mathrm{tm})$ Miniature Wishing Well, Divnick International, Inc., 321 S. Alexander Road, Miamisburg, OH 45342, http://www.divnick.com/ 